\title{
Simplified prediction of the monotonic uniaxial stress-strain curve of non-linear particulate composites
}

\author{
Randoald Mueller, Andreas Mortensen * \\ Ecole Polytechnique Fédérale de Lausanne (EPFL), Laboratory for Mechanical Metallurgy, Materials Institute, MX-D 141, Station 12, \\ CH-1015 Lausanne, Switzerland
}

Received 21 October 2005; received in revised form 4 January 2006; accepted 5 January 2006

Available online 7 March 2006

\begin{abstract}
A variational estimate of the non-linear flow stress of two-phase materials is adapted to simplify predictions of the monotonic uniaxial response of an isotropic particle-reinforced metal. Simplifications to the model are: (i) calculation of the composite elastic and plastic strain is decoupled and (ii) when calculating the composite plastic strain, the reinforcement is taken to be perfectly rigid while the matrix is assumed to deform with no volume change according to a Hollomon power law. This simplified scheme yields analytical expressions that show good agreement with predictions of the full variational estimate, particularly if the Mori-Tanaka or the Torquato identical hard spheres models are used to predict the composite linear elastic modulus. More specifically, error introduced by the above assumptions is significantly less than the difference made by the choice of the appropriate elastic modulus prediction scheme. Use of the approach proposed here is thus justified in practical applications, given the considerable simplification they bring to the calculation. (c) 2006 Acta Materialia Inc. Published by Elsevier Ltd. All rights reserved.
\end{abstract}

Keywords: Particulate-reinforced composites; Elastic behaviour; Plastic deformation; Mean field analysis; Secant moduli method

\section{Introduction}

The mechanical behaviour of composites combining a ductile elastoplastic matrix with discrete particles or fibres of a stiffer elastic reinforcing phase has been the subject of a considerable body of research, summarized in several reviews [1-7]. To predict the stress-strain relation in such composites knowing their constituent phases, two approaches dominate: (i) numerical, typically finite-element, models and (ii) analytical effective-medium schemes.

Numerical methods give essentially unrestricted predictions of the mechanical behaviour of more or less large unit cells, taken to be representative of the composite. Being precise for the periodic microstructure they assume, results from numerical simulation of composite deformation are often used as benchmark tests of more general theories of composite deformation. Their disadvantage is that, to be

\footnotetext{
${ }^{*}$ Corresponding author. Tel.: +412169329 12; fax: +41216934664.

E-mail address: andreas.mortensen@epfl.ch (A. Mortensen).
}

representative of realistic microstructures, the required cell size may need to be large and hence demanding in computing power. In recent work, it was shown that this limitation is not very stringent for fully linear elastic deformation; however, the question remains open for elastoplastic deformation [8-13]. This limitation is, furthermore, significantly worsened if the composites contain larger-scale microstructural features such as reinforcement clustering or sporadic internal damage: in these cases, present computing power is often insufficient if truly representative three-dimensional structures are to be modelled.

Analytical approaches to composite deformation are less specific and hence less precise than numerical models; their aim is rather to provide general, theory-based and directly usable predictions of composite deformation knowing broad features of the composite microstructure, such as the reinforcement shape and orientation. Given the complexity of the problem at hand, analytical schemes rest, of necessity, on approximations. When predicting the monotonic flow stress of non-linear composites using 
analytical mean-field methods, two main approximations are generally invoked in the calculation.

The first is to assimilate the prediction of a non-linear matrix composite flow stress to that of the elastic stiffness tensor of a linear elastic composite. Examples include: (i) assimilation of the average matrix plastic strain to a uniform eigenstrain in the corresponding linear elastic composite (the classic "Eshelby" elastoplastic calculation, summarized, for example, in Ref. [1]), (ii) assimilation of the matrix to a linear elastic medium having the tangent modulus of the matrix at a defined point of matrix deformation (tangent modulus methods), or (iii) assimilation of the matrix to a linear elastic medium having a modulus defined by the ratio of stress to strain at a defined point of matrix deformation (secant modulus approximations). This step of the derivation has now received a rigorous basis based on variational approaches.

The second approximation that is generally made in analytical mean-field schemes follows from the first, in that it is needed to predict the elastic moduli of linear composites containing more than a few volume per cent reinforcement. Approximations that have most often been used to this end for non-dilute two-phase linear elastic composites include (i) the Mori-Tanaka model, corresponding to the lower Hashin-Shtrikman bound for isotropic composites with a spherical reinforcement (e.g., Refs. [3,4,12,14-16]), (ii) the two-phase self-consistent model (e.g., Refs. $[15,17,18])$, (iii) the generalized self-consistent scheme (e.g., Refs. [3,19-21]), and (iv) the more recent third-order approximation models of Torquato [12].

Analytical calculation schemes for non-linear composite deformation have seen significant progress over the past 10 years. The non-linear variational estimates proposed by Ponte Castañeda [4,22-27], shown to be equivalent to a particular secant modulus approximation (generally designated as the "modified" secant modulus) [4,19,21,28], match with impressive accuracy predictions of corresponding finite-element simulations for monotonic loading of non-linear composites $[4,11,12,16,29-32]$ (for specific illustrations of this point, see Figs. 3, 4, 6, and 11 of Ref. [3], Figs. 4 and 5 of Ref. [12], Fig. 2(b) of Ref. [16], Fig. 1 of Ref. [31], Figs. 10 and 11 of Ref. [4], and Figs. 4 and 5 of Ref. [33]). These recent advances, however, unfortunately come at a price: the models are not straightforward in their implementation, the equations being somewhat unwieldy and generally requiring numerical schemes for their solution, even in relatively simple situations.

Our aim in this article is to make the variational estimate, or equivalently the modified secant moduli model, for non-linear composite deformation somewhat more user-friendly, by directly building on the work of Ponte Castañeda and Suquet. We base what follows on three observations:

1. All analytical models, including the more recent variational estimates, can only aim for limited precision since (i) they rest on an analytical estimation of the composite elastic modulus, (ii) there is an inherent variability in the mechanical behaviour of "real" composites, and (iii) other assumptions (such as simplification of the shape of the reinforcement) will always carry a cost in terms of precision.

2. The expressions are much simpler if one focuses on simple constituent stress-strain relations such as power-law hardening, as already pointed out by Suquet and Ponte Castañeda [3,4,19,21,24,29].

3. Nearly all experimental investigations of composite behaviour deal with monotonic uniaxial deformation; we therefore focus on this case exclusively.

Given that many elastoplastic composites combine a ductile matrix with equiaxed particles that are generally assimilated to spheres (in both finite-element and analytical mean-field approaches), we also restrict our attention to this case only. Extension of the scheme to tackle a wider range of microstructures poses no fundamental difficulty, being mainly a matter of accessing the relevant composite linear elastic deformation model. For instance, many inclusion types can be modelled as ellipsoids, either aligned or randomly oriented; for this case, relevant expressions already exist $[25,26,34-36]$.

In what follows, predictions from the variational method of Ponte Castañeda and the equivalent "modified" secant modulus model are first briefly discussed with a focus on the monotonic uniaxial stress-strain law of elastoplastic matrix composites reinforced with stiff isolated spheres. Specific simplifications are then tested. Results show that these assumptions only carry a small cost in terms of precision, while simplifying the equations considerably.

\section{Variational (or "modified secant model") prediction}

\subsection{General}

The variational estimate of non-linear composite flow stress [4,22-27,29] in effect extends mean-field schemes for composite elasticity to non-linear matrix behaviour by approximating the matrix with a linear-elastic material having a variationally optimized modulus for the relevant point of matrix deformation history within the composite. This approach has in particular been formulated for metal matrix composites, i.e., structures containing elastic reinforcements within a softer isotropic elastoplastic power-law hardening matrix $[4,19,25-27,35]$. Given its equivalence with the secant method using the quadratic average (second-order moment) of the strain over each phase $[21,28]$ (as opposed to using the average phase strain, which generally gives overly stiff predictions and suffers important limitations $[3,4,16,37])$, formulations of this model also exist in the form of secant modulus estimates $[14,15,18,20,28,37,38]$.

We consider in what follows a composite that can be modelled as randomly distributed spheres of a stiff linear isotropic elastic reinforcing phase (subscript $r$ ) distributed 
in an isotropic elastoplastic matrix (subscript m). Matrix plastic flow is assumed to follow the von Mises yield criterion with isotropic power-law hardening:

$\sigma_{\mathrm{eq}}=c \cdot \varepsilon_{\mathrm{eq}}^{n}$

where $\varepsilon_{\mathrm{eq}}$ is the equivalent plastic strain, $c$ is the strength coefficient, $n$ is the hardening exponent, and $\sigma_{\mathrm{eq}}$ is the equivalent stress of the matrix material. Adding the elastic and plastic strains, the matrix uniaxial (tension/compression) stress-strain curve is then described by the Ramberg-Osgood relation:

$\varepsilon=\frac{\sigma}{E_{\mathrm{m}}}+\left(\frac{\sigma}{c}\right)^{1 / n}$

where $\varepsilon$ is the tensile strain and $\sigma$ is the tensile stress of the matrix material, and $E_{\mathrm{m}}$ is its Young's modulus.

Calculation of the composite uniaxial monotonic stressstrain curve according to the scheme involves two main steps: (i) calculation of the elastic moduli of the corresponding isotropic non-dilute fully linear elastic composite and (ii) assimilation of the same composite under nonlinear deformation to a linear (secant modulus) composite. We examine these in turn.

\subsection{The linear problem}

Five among the more usual schemes for the estimation of the elastic modulus of linear elastic composites are compared here:

(i) the Mori-Tanaka (MT) model;

(ii) the two-phase self-consistent (SC) model;

(iii) the differential effective-medium (DEM) approximation;

(iv) the generalized self-consistent (GSC) scheme;

(v) the Torquato identical hard spheres (TIHS) approximation, one of the third-order approximation models proposed by Torquato.

These models predict the bulk modulus $K_{\mathrm{c}}$ and the shear modulus $G_{\mathrm{c}}$ of a linear elastic isotropic spherical particlereinforced composite as a function of the bulk and the shear moduli of the constituents: $K_{\mathrm{m}}$ and $G_{\mathrm{m}}$ for the matrix, $K_{\mathrm{r}}$ and $G_{\mathrm{r}}$ for the reinforcement, knowing the volume fraction of the reinforcement $V_{\mathrm{r}}$. All materials (matrix, reinforcement, and composite) being assumed isotropic, knowing $K_{x}$ and $G_{x}$, the Young's modulus $E_{x}$ and the Poisson ratio $v_{x}$ of material $x$ are given by

$E_{x}=\frac{9 K_{x}}{1+\frac{3 K_{x}}{G_{x}}}$

$v_{x}=\frac{1-\frac{2 G_{x}}{3 K_{x}}}{2+\frac{2 G_{x}}{3 K_{x}}}$

where index $x$ is $\mathrm{c}, \mathrm{m}$, or $\mathrm{r}$ for the composite, the matrix, and the reinforcing particle phase, respectively. Equations for each model are listed below. (i) The MT model. Mori and Tanaka developed an "average stress" concept to extend Eshelby's equivalent inclusion method to composites in which the volume fraction of ellipsoidal inclusions is large [39]. For an isotropic two-phase composite containing spherical particles, composite bulk and shear moduli are [40]

$\frac{K_{\mathrm{c}}}{K_{\mathrm{m}}}=1+\frac{V_{\mathrm{r}}\left(K_{\mathrm{r}}-K_{\mathrm{m}}\right)}{\alpha_{\mathrm{m}}\left(1-V_{\mathrm{r}}\right)\left(K_{\mathrm{r}}-K_{\mathrm{m}}\right)+K_{\mathrm{m}}} \quad$ with $\quad \alpha_{\mathrm{m}}=\frac{3 K_{\mathrm{m}}}{3 K_{\mathrm{m}}+4 G_{\mathrm{m}}}$

$\frac{G_{\mathrm{c}}}{G_{\mathrm{m}}}=1+\frac{V_{\mathrm{r}}\left(G_{\mathrm{r}}-G_{\mathrm{m}}\right)}{\beta_{\mathrm{m}}\left(1-V_{\mathrm{r}}\right)\left(G_{\mathrm{r}}-G_{\mathrm{m}}\right)+G_{\mathrm{m}}} \quad$ with $\quad \beta_{\mathrm{m}}=\frac{6}{5} \frac{K_{\mathrm{m}}+2 G_{\mathrm{m}}}{3 K_{\mathrm{m}}+4 G_{\mathrm{m}}}$

These moduli correspond to the Hashin-Shtrikman lower bound if the matrix is the soft phase and coincide with the upper bound if the matrix is the hard phase [41]. As such, they correspond to the softest isotropic random two-phase composite one may envisage, of hard (generally polydisperse) spheres uniformly separated by a layer of the softer matrix.

(ii) The SC model. Initially the self-consistent model was derived to approximate the elastic behaviour of polycrystals; however, it is also used for composites [42]. It considers the reference material to be the composite itself and the effective moduli are obtained self-consistently. If the inclusions are spheres distributed such that the composite is statistically isotropic overall, the effective elastic moduli are determined by [43]

$\frac{1-V_{\mathrm{r}}}{K_{\mathrm{c}}-K_{\mathrm{r}}}+\frac{V_{\mathrm{r}}}{K_{\mathrm{c}}-K_{\mathrm{m}}}=\frac{\alpha}{K_{\mathrm{c}}} \quad$ with $\quad \alpha=\frac{K_{\mathrm{c}}}{K_{\mathrm{c}}+\frac{4}{3} G_{\mathrm{c}}}$

$\frac{1-V_{\mathrm{r}}}{G_{\mathrm{c}}-G_{\mathrm{r}}}+\frac{V_{\mathrm{r}}}{G_{\mathrm{c}}-G_{\mathrm{m}}}=\frac{\beta}{G_{\mathrm{c}}} \quad$ with $\quad \beta=\frac{3-\alpha}{5}$

Although it is derived starting with equations relevant to isolated spheres distributed in a continuous matrix, this model is generally considered to be most appropriate for composites in which both phases are interconnected. The reason for this is that, as the reinforcement volume fraction increases, the self-consistent prediction rapidly exceeds the dilute limit, tending to very high modulus values above roughly $30 \mathrm{vol} . \%$ reinforcement.

(iii) The DEM approximation. In this model, the reinforcement is incrementally added to the composite, using the dilute solution for each increment and integrating equations to reach a finite volume fraction of reinforcement [44]. The following differential equations are obtained in the case of a particle-reinforced composite [34]:

$$
\begin{aligned}
& \left(1-V_{\mathrm{r}}\right) \frac{\partial K_{\mathrm{c}}}{\partial V_{\mathrm{r}}}=\left(K_{\mathrm{c}}+\frac{4}{3} G_{\mathrm{c}}\right) \frac{K_{\mathrm{r}}-K_{\mathrm{c}}}{K_{\mathrm{r}}+\frac{4}{3} G_{\mathrm{c}}} \\
& \left(1-V_{\mathrm{r}}\right) \frac{\partial G_{\mathrm{c}}}{\partial V_{\mathrm{r}}}=\left(K_{\mathrm{c}}+H\right) \frac{G_{\mathrm{r}}-G_{\mathrm{c}}}{G_{\mathrm{r}}+H} \quad \text { with } \\
& H=\frac{G_{\mathrm{c}}\left(\frac{3}{2} K_{\mathrm{c}}+\frac{4}{3} G_{\mathrm{c}}\right)}{K_{\mathrm{c}}+2 G_{\mathrm{c}}}
\end{aligned}
$$


Given its starting assumption, this derivation is a priori best suited for composites containing a wide range of particle radii (where larger particles can be viewed as embedded in a "continuum" composite of matrix and smaller particles). It yields, for stiff particles in a softer matrix, predictions that are intermediate between the MT and SC schemes, as one would expect.

(iv) The GSC scheme. Christensen developed a model involving three phases: a spherical reinforcement inclusion, a spherical shell of matrix material with the appropriate matrix/reinforcement volume fraction ratio, both surrounded by an infinite outer region of equivalent homogeneous material of unlimited extent having the properties of the composite. The final equations are [45-47]

$$
\begin{gathered}
K_{\mathrm{c}}=K_{\mathrm{m}}+\frac{V_{\mathrm{r}}\left(K_{\mathrm{r}}-K_{\mathrm{m}}\right)}{1+\left(1-V_{\mathrm{r}}\right) \frac{K_{\mathrm{r}}-K_{\mathrm{m}}}{K_{\mathrm{m}}+\frac{4}{3} G_{\mathrm{m}}}} \\
\left(\frac{G_{\mathrm{c}}}{G_{\mathrm{m}}}\right)^{2} \cdot A+\frac{G_{\mathrm{c}}}{G_{\mathrm{m}}} \cdot B+D=0
\end{gathered}
$$

with

$$
\begin{aligned}
A= & 8\left[\frac{G_{\mathrm{r}}}{G_{\mathrm{m}}}-1\right]\left(4-5 v_{\mathrm{m}}\right) \eta_{1} \cdot V_{\mathrm{r}}^{10 / 3}-2\left[63\left(\frac{G_{\mathrm{r}}}{G_{\mathrm{m}}}-1\right) \eta_{2}\right. \\
& \left.+2 \eta_{1} \eta_{3}\right] \cdot V_{\mathrm{r}}^{7 / 3}+252\left[\frac{G_{\mathrm{r}}}{G_{\mathrm{m}}}-1\right] \eta_{2} \cdot V_{\mathrm{r}}^{5 / 3}-50\left[\frac{G_{\mathrm{r}}}{G_{\mathrm{m}}}-1\right] \\
& \times\left(7-12 v_{\mathrm{m}}+8 v_{\mathrm{m}}^{2}\right) \eta_{2} \cdot V_{\mathrm{r}}+4\left(7-10 v_{\mathrm{m}}\right) \eta_{2} \eta_{3}
\end{aligned}
$$

This model is viewed as an improvement on the Mori-Tanaka calculation that better reproduces the distribution of phases and stress-strain fields in a composite of particles separated by matrix.

(v) The TIHS approximation. Torquato developed estimates for the effective elastic moduli of two-phase isotropic dispersions by truncating exact series expansions that determine the effective stiffness tensor after third-order terms $[48,49]$. These third-order series expressions, given as a function of two microstructural parameters $\zeta_{\mathrm{r}}$ and $\eta_{\mathrm{r}}$, provide composite modulus estimates for a wide range of phase moduli, for various non-clustered sphere dispersions and volume fractions. Interpenetration of the dispersed spheres constituting the inclusion phase is not permitted in this "hard-sphere" approximation; hence, the monomodal reinforcement content cannot exceed the volume fraction of densely random packed spheres, namely 64\%. The following equations (Eqs. (13) and (14)) are given in [34]:

$$
\begin{aligned}
& K_{\mathrm{c}}=\frac{K_{\mathrm{m}}+\frac{4}{3} G_{\mathrm{m}} \kappa_{\mathrm{em}}}{1-\kappa_{\mathrm{em}}} \\
& G_{\mathrm{c}}=\frac{1}{1-\mu_{\mathrm{em}}}\left[G_{\mathrm{m}}+\frac{G_{\mathrm{m}} \mu_{\mathrm{em}}\left(\frac{3}{2} K_{\mathrm{m}}+\frac{4}{3} G_{\mathrm{m}}\right)}{K_{\mathrm{m}}+2 G_{\mathrm{m}}}\right]
\end{aligned}
$$

where

$$
\begin{aligned}
& \kappa_{\mathrm{em}}=\frac{V_{\mathrm{r}} \kappa_{\mathrm{rm}}}{1-\frac{10 G_{\mathrm{m}} \kappa_{\mathrm{rm}} \mu_{\mathrm{m}} \zeta_{\mathrm{r}}}{3\left(K_{\mathrm{m}}+2 G_{\mathrm{m}}\right)}\left(1-V_{\mathrm{r}}\right)} \\
& \mu_{\mathrm{em}}=\frac{V_{\mathrm{r}} \mu_{\mathrm{rm}}}{1-\frac{2 G_{\mathrm{m}} \kappa_{\mathrm{rm}} \mu_{\mathrm{rm}} \zeta_{\mathrm{r}}}{3\left(K_{\mathrm{m}}+2 G_{\mathrm{m}}\right)}\left(1-V_{\mathrm{r}}\right)-\frac{5 G_{\mathrm{m}}\left(2 K_{\mathrm{m}}+3 G_{\mathrm{m}}\right) \mu_{\mathrm{rm}}^{2} \zeta_{\mathrm{r}}}{6\left(K_{\mathrm{m}}+2 G_{\mathrm{m}}\right)^{2}}\left(1-V_{\mathrm{r}}\right)-\left(\frac{3 K_{\mathrm{m}}+G_{\mathrm{m}}}{K_{\mathrm{m}}+2 G_{\mathrm{m}}}\right)^{2} \frac{\mu_{\mathrm{rm}}^{2} \eta_{\mathrm{r}}}{6}\left(1-V_{\mathrm{r}}\right)}
\end{aligned}
$$

$$
\begin{aligned}
B= & -4\left[\frac{G_{\mathrm{r}}}{G_{\mathrm{m}}}-1\right]\left(1-5 v_{\mathrm{m}}\right) \eta_{1} \cdot V_{\mathrm{r}}^{10 / 3}+4\left[63\left(\frac{G_{\mathrm{r}}}{G_{\mathrm{m}}}-1\right) \eta_{2}\right. \\
& \left.+2 \eta_{1} \eta_{3}\right] \cdot V_{\mathrm{r}}^{7 / 3}-504\left[\frac{G_{\mathrm{r}}}{G_{\mathrm{m}}}-1\right] \eta_{2} \cdot V_{\mathrm{r}}^{5 / 3}+150\left[\frac{G_{\mathrm{r}}}{G_{\mathrm{m}}}-1\right] \\
& \times\left(3-v_{\mathrm{m}}\right) v_{\mathrm{m}} \eta_{2} \cdot V_{\mathrm{r}}+3\left(15 v_{\mathrm{m}}-7\right) \eta_{2} \eta_{3} \\
D= & 4\left[\frac{G_{\mathrm{r}}}{G_{\mathrm{m}}}-1\right]\left(5 v_{\mathrm{m}}-7\right) \eta_{1} \cdot V_{\mathrm{r}}^{10 / 3}-2\left[63\left(\frac{G_{\mathrm{r}}}{G_{\mathrm{m}}}-1\right) \eta_{2}\right. \\
& \left.+2 \eta_{1} \eta_{3}\right] \cdot V_{\mathrm{r}}^{7 / 3}+252\left[\frac{G_{\mathrm{r}}}{G_{\mathrm{m}}}-1\right] \eta_{2} \cdot V_{\mathrm{r}}^{5 / 3} \\
& +25\left[\frac{G_{\mathrm{r}}}{G_{\mathrm{m}}}-1\right]\left(v_{\mathrm{m}}^{2}-7\right) \eta_{2} \cdot V_{\mathrm{r}}-\left(7+5 v_{\mathrm{m}}\right) \eta_{2} \eta_{3} \\
\eta_{1}= & {\left[\frac{G_{\mathrm{r}}}{G_{\mathrm{m}}}-1\right]\left(49-50 v_{\mathrm{r}} v_{\mathrm{m}}\right)+35 \frac{G_{\mathrm{r}}}{G_{\mathrm{m}}}\left(v_{\mathrm{r}}-2 v_{\mathrm{m}}\right)+35\left(2 v_{\mathrm{r}}-v_{\mathrm{m}}\right) } \\
\eta_{2}= & 5 v_{\mathrm{r}}\left[\frac{G_{\mathrm{r}}}{G_{\mathrm{m}}}-8\right]+7\left[\frac{G_{\mathrm{r}}}{G_{\mathrm{m}}}+4\right] \\
\eta_{3}= & \frac{G_{\mathrm{r}}}{G_{\mathrm{m}}}\left(8-10 v_{\mathrm{m}}\right)+\left(7-5 v_{\mathrm{m}}\right)
\end{aligned}
$$

with

$$
\begin{aligned}
& \kappa_{\mathrm{rm}}=\frac{K_{\mathrm{r}}-K_{\mathrm{m}}}{K_{\mathrm{r}}+\frac{4}{3} G_{\mathrm{m}}} \\
& \mu_{\mathrm{rm}}=\frac{G_{\mathrm{r}}-G_{\mathrm{m}}}{G_{\mathrm{r}}+\frac{G_{\mathrm{m}}\left(\frac{3}{2} K_{\mathrm{m}}+\frac{4}{3} G_{\mathrm{m}}\right)}{K_{\mathrm{m}}+2 G_{\mathrm{m}}}}
\end{aligned}
$$

The values of $\zeta_{\mathrm{r}}$ and $\eta_{\mathrm{r}}$ come respectively from Table 22.1 and Table 22.3 in Ref. [34]. This model is obviously best suited for the microstructure for which it was derived, namely non-interpenetrating monodisperse spheres of one phase distributed in a matrix of the other.

\subsection{Passage from non-linear to linear deformation}

Consider now the composite deformed to stress $\Sigma$. Although the composite stress and strain are always the volumetric averages of average stresses and strains in matrix and reinforcement, respectively, the values of each 
of these quantities in each phase are unknown. So is the average composite strain, $\Xi$.

We use the "dual" formulation, which imposes a constant stress field on the composite, as this is more convenient for uniaxial deformation $[19,27]$. Specifically, we use the equations in Ref. [19], beginning with the relation between the effective matrix secant shear modulus $\left(G_{\mathrm{ms}}\right)$ and the matrix equivalent stress $\sigma_{\text {eq }}$ :

$G_{\mathrm{ms}}=\frac{1}{\frac{1}{G_{\mathrm{m}}}+\frac{3}{\sigma_{\mathrm{eq}}}\left(\frac{\sigma_{\mathrm{eq}}}{c}\right)^{1 / n}}$

where $\sigma_{\mathrm{eq}}$ is estimated as the volumetric average of the second-order moment of the stress field in the linear elastic secant modulus composite material to which the non-linear composite is assimilated. This second-order moment is in turn derived from the relevant linear elasticity expression (e.g., one of the expressions given above) giving the composite linear elastic compliance tensor $\mathbf{M}$ as a function of matrix and reinforcement moduli according to the expression

$\sigma_{\mathrm{eq}}^{2}=\frac{3}{1-V_{\mathrm{r}}}\left[\underline{\Sigma}:\left(\frac{\partial M}{\partial\left(1 / G_{\mathrm{m}}\right)}\right)_{\left(G_{\mathrm{m}}=G_{\mathrm{ms}}\right)}: \underline{\Sigma}\right]$

where $\Sigma$ is the composite (effective) stress tensor $[3,12,38,50]$. In uniaxial (tensile/compressive) deformation, only one composite stress component $(\Sigma)$ is non-zero. Eq. (16) may then be simplified and the composite stress is directly expressed as a function of the matrix equivalent stress $\sigma_{\text {eq }}$ from which $G_{\mathrm{ms}}$ is estimated:

$\Sigma=\sqrt{\frac{1-V_{\mathrm{r}}}{3 a}} \cdot \sigma_{\mathrm{eq}} \quad$ with $\quad a=\left(\frac{\partial\left(1 / E_{\mathrm{c}}\right)}{\partial\left(1 / G_{\mathrm{m}}\right)}\right)_{\left(G_{\mathrm{m}}=G_{\mathrm{ms}}\right)}$

The matrix secant bulk modulus $K_{\mathrm{ms}}$ remains equal to its linear elastic bulk modulus $K_{\mathrm{m}}$ since plastic deformation causes no volume change.

Operationally, the calculation is performed by solving Eq. (15) and then Eq. (17) for a given value of $\sigma_{\text {eq }}$, to deduce $\Sigma$. To this end, numerical methods are used since $a$, defined by the elastic model adopted (see previous section), is not generally given by simple analytical expressions. The present calculations were performed using Mathcad (Mathcad Professional 2001 software, (C) 19862000 Mathsoft, Inc., USA).

One then deduces the total composite uniaxial strain, $\Xi$ :

$\Xi=\frac{\Sigma}{E_{\mathrm{cs}}}$

where $E_{\mathrm{cs}}$ is the secant composite Young's modulus (a function of $V_{\mathrm{r}}, K_{\mathrm{r}}, G_{\mathrm{r}}, K_{\mathrm{m}}$, and $\left.G_{\mathrm{ms}}\right) . \Xi$ is in turn equal to the sum of elastic strain, $\Xi_{\mathrm{el}}=\Sigma / E_{\mathrm{c}}$, and plastic strain:

$\Xi_{\mathrm{pl}}=\frac{\Sigma}{E_{\mathrm{cs}}}-\frac{\Sigma}{E_{\mathrm{c}}}$

Individual stress and strain values in each phase are also directly deduced from the elastic model knowing the instantaneous value of the matrix secant moduli.

\section{Proposed simplification of the method}

\subsection{Assumptions}

The composite flow curve estimation scheme of Ponte Castañeda and Suquet, presented above, is known to provide good agreement with predictions of numerical models; however, as seen above, implementing the calculation is not straightforward. It would therefore be of interest to explore whether, with a few simple yet reasonable assumptions, the method can be adapted at minimal cost in terms of precision so as to ease its implementation significantly. The assumptions we now explore build largely on simplifications already proposed and explored in part by Suquet and Ponte Castañeda [4,19,25-27,29,35].

First, we decouple completely the elastic and the plastic composite strain calculations. We assume that, at given composite stress $\Sigma$, the composite strain $\Xi$ is the sum of:

- the elastic strain $\Xi_{\mathrm{el}}$ of the corresponding fully elastic composite, predicted by the relevant linear elastic modulus model (see Section 2.2) and

- the plastic strain $\Xi_{\mathrm{pl}}$ that would be displayed by a similar composite having (i) an incompressible isotropic von Mises matrix displaying the relevant power-law (Hollomon) stress-strain curve and (ii) rigid inclusions.

With these assumptions, the simplified composite from which the composite plastic strain is estimated is itself also a power-law incompressible von Mises plastic material having the same exponent $n$ as its matrix $[4,19,29]$ :

$\Sigma_{\text {eq }}=C \cdot \Xi_{\text {eq }}^{n}$

where $\Sigma_{\text {eq }}$ and $\Xi_{\text {eq }}$ are the composite von Mises equivalent stress and equivalent strain, respectively. The ratio $C / c$ of composite strength coefficient to matrix strength coefficient is then, for given $n$ and $V_{\mathrm{r}}$, a constant throughout the composite (monotonic proportional) loading history.

Prediction of the composite stress-strain curve is then far simpler, since instead of solving numerically the set of equations given above for each value of the composite stress $\Sigma$, one simply:

1. calculates the composite modulus $E_{\mathrm{c}}$ using the appropriate linear elasticity model (generally one of the five given in Section 2.2);

2. finds the ratio $C / c$ as a function of $n$ and $V_{\mathrm{r}}$ predicted by the method using the same linear elastic model in the calculation but for an incompressible matrix and a rigid reinforcement. As we show below, this last calculation is actually quite simple with the assumptions made above.

\subsection{Calculating the plastic strength ratio $\mathrm{Clc}$}

When the two phases are incompressible $\left(K_{\mathrm{m}} / G_{\mathrm{ms}}=\right.$ $\left.K_{\mathrm{r}} / G_{\mathrm{r}}=\infty\right)$ and the reinforcement is perfectly rigid 
$\left(G_{\mathrm{r}} / G_{\mathrm{ms}}=\infty\right)$, Eqs. (6), (8), (10), (12), and (14) become, respectively, for each of the five linear elasticity composite modulus prediction schemes

MT : $G_{\mathrm{cs}}=\frac{G_{\mathrm{ms}}\left(3 V_{\mathrm{r}}+2\right)}{2\left(1-V_{\mathrm{r}}\right)}$

$\mathrm{SC}: G_{\mathrm{cs}}=\frac{2 G_{\mathrm{ms}}}{2-5 V_{\mathrm{r}}}$

$\mathrm{DEM}: G_{\mathrm{cs}}=\frac{G_{\mathrm{ms}}}{\left(1-V_{\mathrm{r}}\right)^{5 / 2}}$

$\mathrm{GSC}:\left(\frac{G_{\mathrm{cs}}}{G_{\mathrm{ms}}}\right)^{2} \cdot A+\frac{G_{\mathrm{cs}}}{G_{\mathrm{ms}}} \cdot B+D=0$

with

$A=228 V_{\mathrm{r}}^{10 / 3}-1425 V_{\mathrm{r}}^{7 / 3}+2394 V_{\mathrm{r}}^{5 / 3}-1425 V_{\mathrm{r}}+228$

$B=114 V_{\mathrm{r}}^{10 / 3}+2850 V_{\mathrm{r}}^{7 / 3}-4788 V_{\mathrm{r}}^{5 / 3}+1781 V_{\mathrm{r}}+43$

$D=-342 V_{\mathrm{r}}^{10 / 3}-1425 V_{\mathrm{r}}^{7 / 3}+2394 V_{\mathrm{r}}^{5 / 3}-1603 V_{\mathrm{r}}-271$

TIHS : $G_{\mathrm{cs}}=\frac{G_{\mathrm{ms}}(3 \mu+2)}{2(1-\mu)}$

with

$\mu=\frac{4 V_{\mathrm{r}}}{4-3 V_{\mathrm{r}}\left(1-V_{\mathrm{r}}\right)}$

Moreover, the composite secant Young's modulus is now simply

$E_{\mathrm{cs}}=3 G_{\mathrm{cs}}$

Eq. (15) is also simplified as

$G_{\mathrm{ms}}=\frac{\sigma_{\mathrm{eq}}}{3}\left(\frac{c}{\sigma_{\mathrm{eq}}}\right)^{1 / n}$

Analytical expressions can then be deduced [22] giving directly the ratio between the composite flow stress and the matrix flow stress, $C / c$. The calculation is illustrated in Appendix A, taking as an example the MT scheme. The final expressions are

MT $: \frac{C}{c}=\frac{\left(\frac{3}{2} V_{\mathrm{r}}+1\right)^{(n+1) / 2}}{\left(1-V_{\mathrm{r}}\right)^{n}}$

$\mathrm{SC}: \frac{C}{c}=\left(\frac{2}{2-5 V_{\mathrm{r}}}\right)^{(n+1) / 2} \cdot\left(1-V_{\mathrm{r}}\right)^{(1-n) / 2}$

(corresponding to Eq. (5.15) of Ref. [22])

DEM : $\frac{C}{c}=\frac{1}{\left(1-V_{\mathrm{r}}\right)^{(7 n+3) / 4}}$

(corresponding to Eq. (5.17) of Ref. [22])

GSC $: \frac{C}{c}=\alpha^{(n+1) / 2} \cdot\left(1-V_{\mathrm{r}}\right)^{(1-n) / 2}$

with

$\alpha=\frac{-B+\sqrt{B^{2}-4 A D}}{2 A}$

(where $A, B$, and $D$ are given by Eq. (24))
TIHS $: \frac{C}{c}=\left(\frac{\frac{3}{2} \mu+1}{1-\mu}\right)^{(n+1) / 2} \cdot\left(1-V_{\mathrm{r}}\right)^{(1-n) / 2}$

(where $\mu$ is given by Eq. (25)).

\subsection{Predicting the composite flow curve from that of its constituents according to the simplified scheme}

With the assumptions presented in Section 3.1, the relation between the composite flow stress $\Sigma$ and the composite strain $\Xi$ is given by

$\Xi=\frac{\Sigma}{E_{\mathrm{c}}}+\left(\frac{\Sigma}{C}\right)^{1 / n}$

where expressions for $E_{\mathrm{c}}$ and $C$ are given above for each of the five investigated composite linear elasticity modulus calculation schemes (Eqs. (5)-(14) introduced in Eq. (3) for $E_{\mathrm{c}}$, and Eqs. (28)-(32) for $C$ ).

\section{Results and discussion}

We take as a "practical case study" that of composites combining an aluminium-based matrix $\left(K_{\mathrm{m}}=76 \mathrm{GPa}\right.$, $G_{\mathrm{m}}=26 \mathrm{GPa}$ ) with one of the following two reinforcements:

(i) spherical alumina particles $\left(K_{\mathrm{r}}=238 \mathrm{GPa}, G_{\mathrm{r}}=164\right.$ $\mathrm{GPa})$, a classic reinforcement in metal matrix composites, and

(ii) purely elastic spherical regions having the same elastic moduli as the aluminium matrix. This second case is representative of alloys combining ductile and elastic phases that have a significant fraction of the same metal atoms in common, as is often the case in metallurgy (intermetallic particles in a base metal solid solution or dual-phase steels are classic examples).

We vary the hardening exponent of the matrix, exploring the values $n=0$ (perfectly plastic behaviour), $n=0.1$, and $n=0.2$. The matrix strength coefficient is fixed at an arbitrary but realistic value $c=250 \mathrm{MPa}$. We first examine predictions concerning elastic modulus values, and turn next to elastoplastic composite deformation.

Fig. 1 compares the various predictions for the alumina/ aluminium composite Young's modulus $E_{\mathrm{c}}$. As should be, the lowest among these curves is given by the HashinShtrikman lower bound, corresponding also to the MT model. Interestingly, the TIHS and the GSC models yield relatively similar predictions. The conclusion made in Refs. [51,52], that the GSC model yields accurate predictions of the Young's modulus of alumina particle-reinforced aluminium composites of high volume fraction reinforcement, thus also extends to the TIHS model. As is well known, the SC approximation yields the stiffest result among these five predictions. It is therefore generally considered that this model should only be used when there is significant 


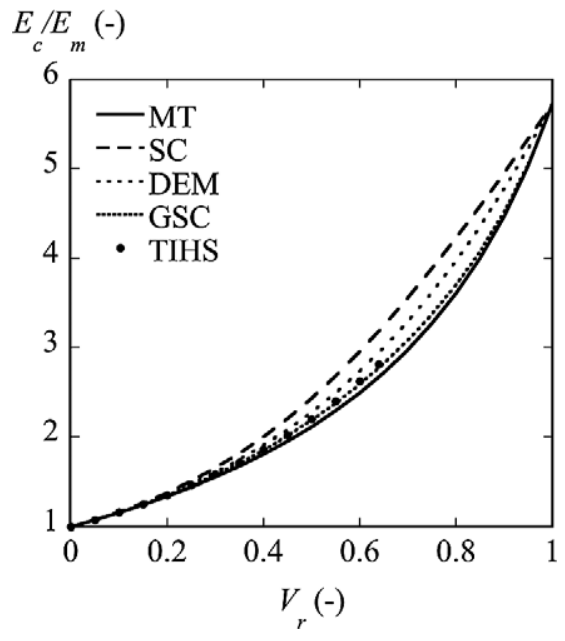

Fig. 1. Scaled Young's modulus $E_{\mathrm{c}} / E_{\mathrm{m}}$ versus $V_{\mathrm{r}}$ predicted by five common effective-medium schemes for aluminium reinforced with spherical alumina particles.

percolation of the reinforcing phase (in which case, however, the Torquato identical interpenetrating spheres model should be considered) [3,34]. The DEM model is intermediate between the TIHS and the SC models.

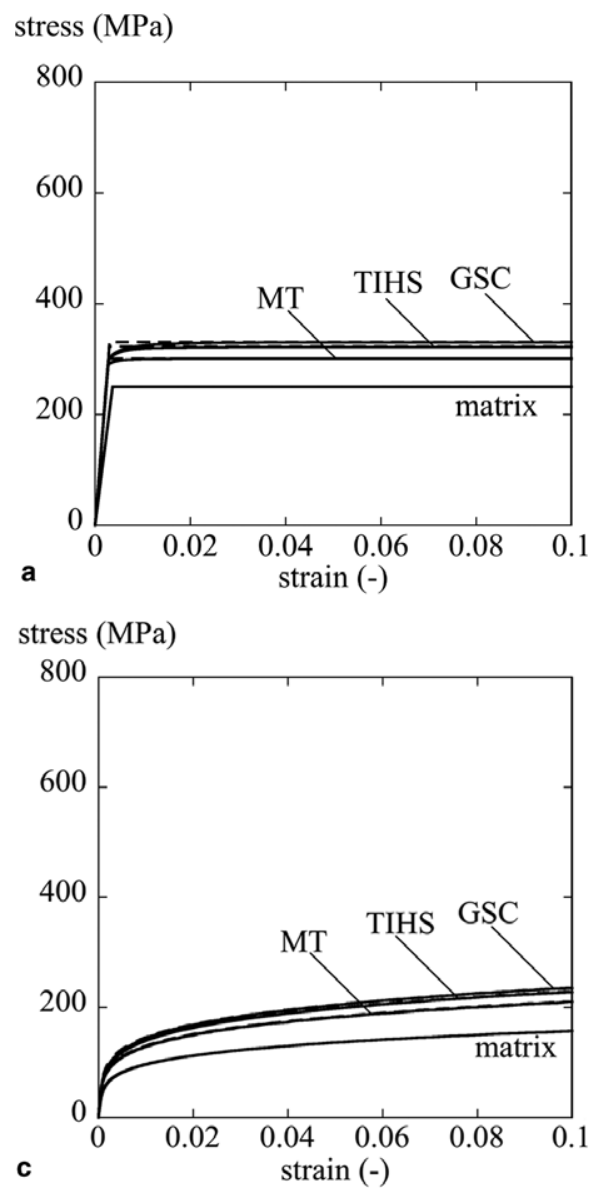

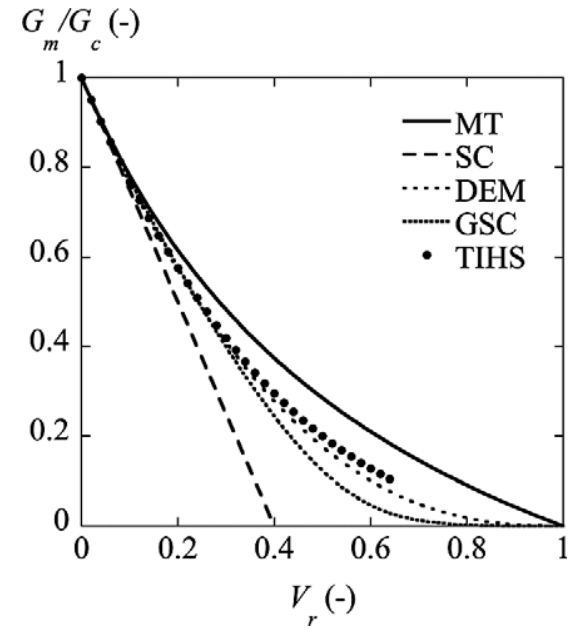

Fig. 2. Inverse of scaled shear modulus $G_{\mathrm{c}} / G_{\mathrm{m}}$ versus $V_{\mathrm{r}}$ predicted by five common effective-medium schemes for an incompressible matrix reinforced with perfectly rigid spheres.

It is interesting to examine the elastic modulus predictions for the case where the reinforcement is rigid and the matrix incompressible, as assumed in the simplified calculation presented above for the prediction of non-linear

stress (MPa)

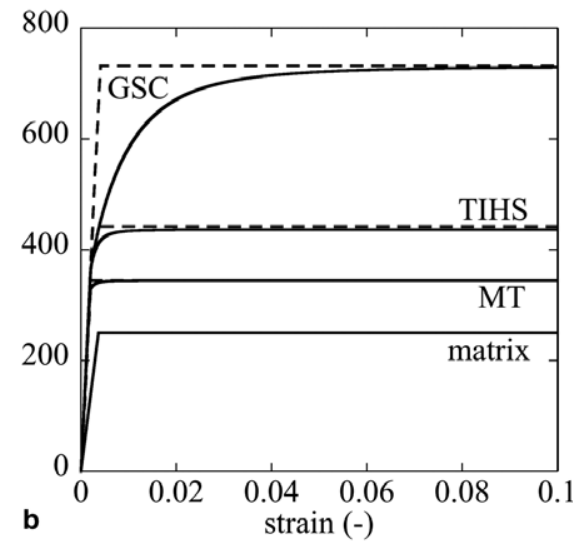

stress $(\mathrm{MPa})$

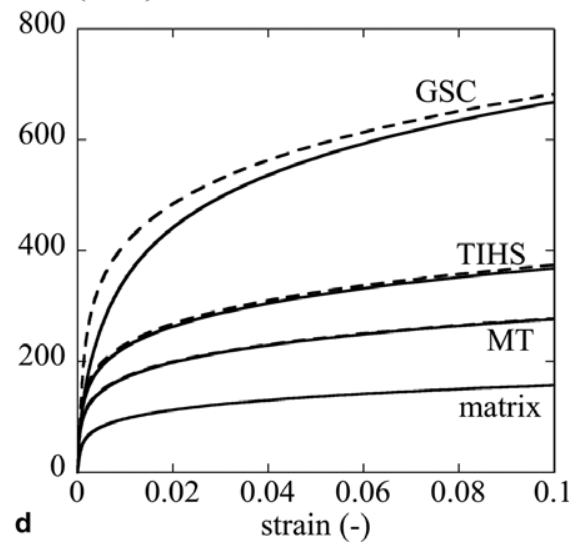

Fig. 3. Typical $\mathrm{Al}-\mathrm{Al}_{2} \mathrm{O}_{3}$ flow curves calculated with Eq. (18) (solid lines) and with the simplified scheme of Eq. (33) (dashed lines) for three effectivemedium elasticity models: (a) $V_{\mathrm{r}}=0.3, n=0$; (b) $V_{\mathrm{r}}=0.6, n=0$; (c) $V_{\mathrm{r}}=0.3, n=0.2$; (d) $V_{\mathrm{r}}=0.6, n=0.2$. 
composite deformation. We plot, instead of the ratio of composite to matrix Young's modulus, the ratio of matrix to composite shear modulus, $G_{\mathrm{m}} / G_{\mathrm{c}}$ (Fig. 2) (to find whether this ratio reaches zero). As known, a percolation threshold is predicted by Eq. (22) at $V_{\mathrm{r}}=0.4$ for the SC model. More interesting is the behaviour of the GSC scheme. This model, similar to the TIHS approximation for a "real" material (Fig. 1), becomes very stiff when the assumption of matrix incompressibility is made (Fig. 2). As mentioned above, two hypotheses are made in plotting Fig. 2: (i) the reinforcing inclusions are perfectly rigid and (ii) the matrix is incompressible. Segurado et al. showed that, making only the first assumption, these two models still yield similar predictions (with $v_{\mathrm{r}}=0.25$ ) [10]. Thus, it is the latter hypothesis that causes this a priori unexpected shift in the position of the GSC model prediction among the five surveyed here, causing it to veer towards the very stiff SC model. This effect of matrix incompressibility on predictions of the GSC method was already observed and discussed by Christensen [47].

Fig. 3 compares predicted stress-strain curves according to the two calculations methods presented in Sections 2 and 3 (the rigorous method and its simplified version presented here) for the $\mathrm{Al}-\mathrm{Al}_{2} \mathrm{O}_{3}$ composite. We explore deliberately high reinforcement volume fractions, as this maximizes differences between the composite and the matrix stress-strain curves on the one hand, and the difference between predictions of the various elasticity models on the other. We focus on three elasticity models, namely the MT, TIHS, and GSC calculation schemes.

Fig. 3 shows that for all three models, apart from a small strain range near the onset of yield, the simplified calculation remains quite close to the predictions of the rigorous approach. Even for large volume fraction of stiff reinforcement (Fig. 3(b) and (d)), the flow curves are mostly governed by the chosen elasticity model and differ far less between the two calculations.

Comparing the three elasticity models it is seen that, while the MT and the TIHS models are relatively close and are well reproduced by the simplified model presented here, the GSC scheme yields significantly higher flow stresses, together with a somewhat greater discrepancy between the simplified and the rigorous secant model predictions. As mentioned above, this can be attributed to the fact that the GSC scheme shifts significantly in its predictions when the matrix becomes incompressible (Fig. 2). The choice of $n$ influences greatly the appearance of the flow curves, but not the accuracy of the simplified calculation procedure.
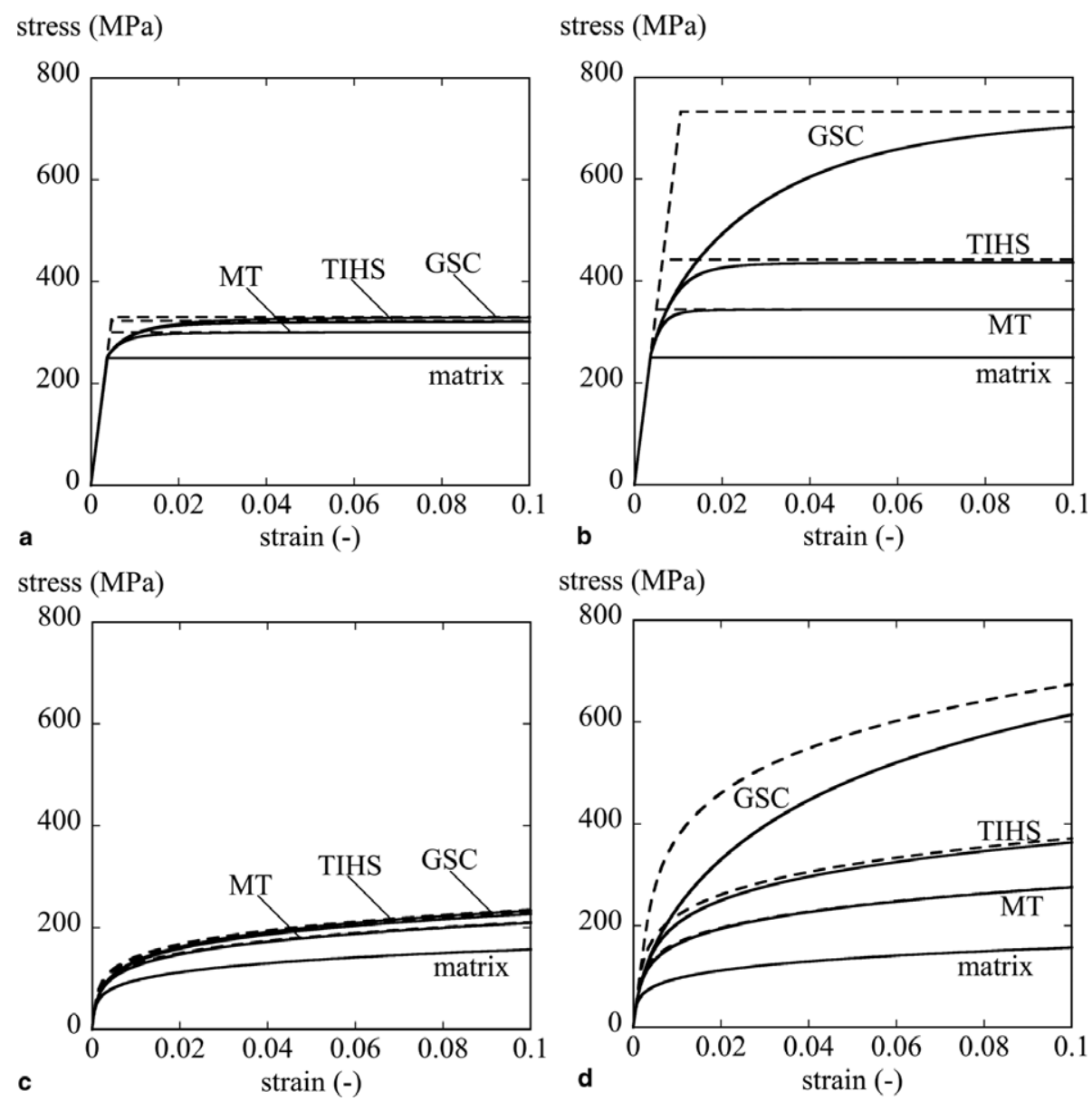

stress (MPa)

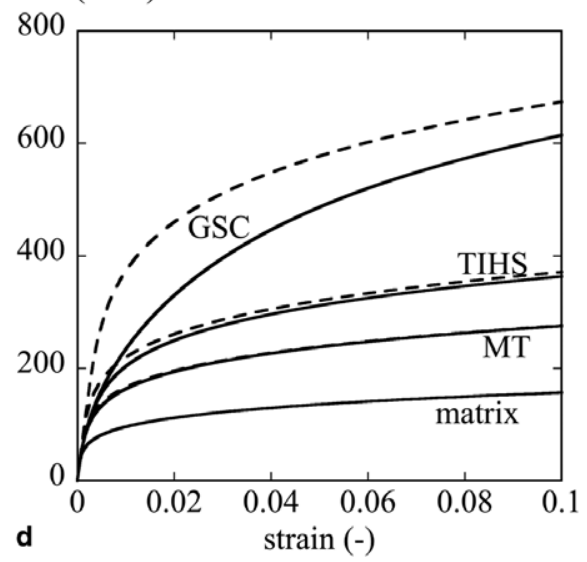

Fig. 4. Typical Al-Al flow curve calculated with Eq. (18) (solid lines) and with the simplified scheme predictions of Eq. (33) (dashed lines) for three effective-medium elasticity models: (a) $V_{\mathrm{r}}=0.3, n=0$; (b) $V_{\mathrm{r}}=0.6, n=0$; (c) $V_{\mathrm{r}}=0.3, n=0.2$; (d) $V_{\mathrm{r}}=0.6, n=0.2$. 
Predictions for the (elastic aluminium)/(elastoplastic aluminium) material are compared in Fig. 4. The curves have a similar aspect to those in Fig. 3 and the simplified and the full calculations remain equally close for both the MT and the TIHS models: the reinforcement stiffness is thus not an essential parameter in the comparison. For the GSC model, however, the simplified scheme yields rather imprecise results: the simplified and the rigorous model converge only at a strain value greater than 0.1 . The reason for the greater difference is the more significant level of elastic strain in the composite: note that the predictions of the simplified model are roughly equivalent at strains near 0.1 for both types of composite (Figs. 3 and $4(\mathrm{~d})$ ), whereas the full calculation yields a rather different result. The stiffer the reinforcement becomes, the lower is the composite elastic strain and the closer the prediction of the simplified model comes to that of the full calculation. In other words, predictions of the present approximate scheme are increasingly accurate as the reinforcement is stiffer in relation to the matrix, a fact that could easily be guessed if one re-examines the initial assumptions made.

Figs. 5 and 6 compare the predicted values of the ratio of the two-phase material flow stress to the matrix flow stress at a fixed strain, namely 0.02 (a realistic value for most particle-reinforced ductile matrix composites). Fig. 5 is for the MT scheme, Fig. 6 for the TIHS model; each has one plot for each of the two reinforcements considered here: $\mathrm{Al}_{2} \mathrm{O}_{3}$ (a, left) and elastic $\mathrm{Al}$ (b, right). As seen, agreement between the simplified and the full calculations remains good for all values of matrix hardening exponent $n$ and inclusion volume fraction $V_{\mathrm{r}}$ explored here
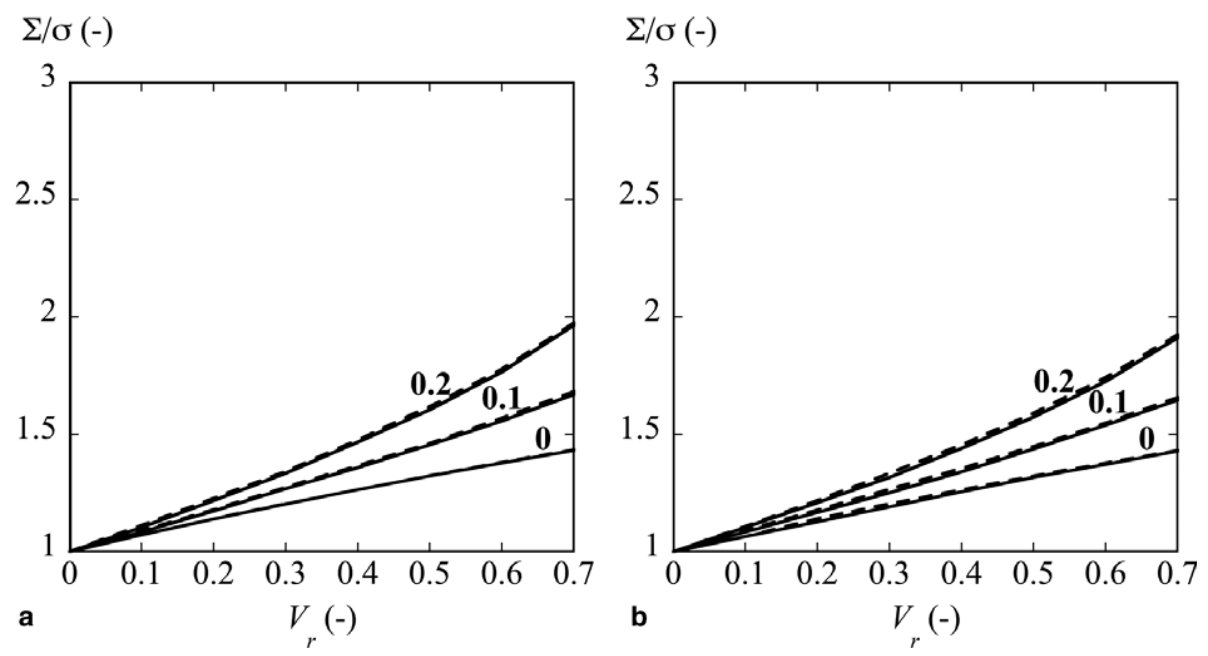

Fig. 5. Normalized composite flow stress at $2 \%$ composite uniaxial deformation as a function of the reinforcement volume fraction predicted by the full scheme (solid lines), and by the simplified scheme (dashed lines) for different $n$ values $(0-0.2)$ with, in both cases, the $\mathrm{MT}$ model: (a) $\mathrm{Al}-\mathrm{Al}_{2} \mathrm{O}_{3}$ system; (b) Al-Al system.
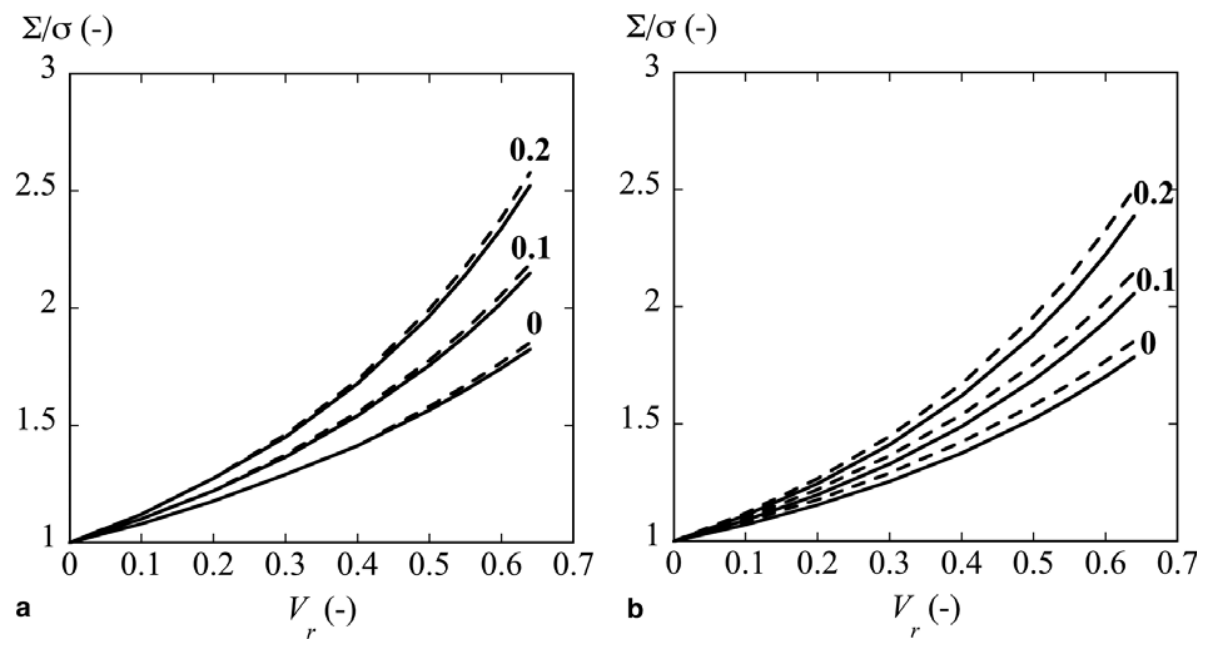

Fig. 6. Normalized composite flow stress at $2 \%$ composite uniaxial deformation as a function of the reinforcement volume fraction predicted by the full scheme (solid lines), and by the simplified scheme (dashed lines) for different $n$ values $(0-0.2)$ with, in both cases, the TIHS approximation: (a) $\mathrm{Al}_{-} \mathrm{Al}_{2} \mathrm{O}_{3}$ system; (b) Al-Al system. 
(including for highly reinforced composites). With a stiff reinforcement (Figs. 5 and 6(a)), the curves nearly overlap; with the elastically homogeneous material, the curves remain within $10 \%$ of one another (Figs. 5 and $6($ b)). In both cases, for given $n$ and $V_{\mathrm{r}}$, the difference that exists between predictions of the simplified and the full models remains well below the difference made by the choice of elastic modulus prediction scheme: compare Fig. 5(a) with Fig. 6(a), and Fig. 5(b) with Fig. 6(b), respectively.

It is, in conclusion, this last choice, namely of the appropriate elastic modulus calculation model, that has by far the greatest influence on the variational (or "modified" secant modulus) prediction of the composite non-linear uniaxial stress-strain curve, a fact that has already been pointed out $[3,4,29,32]$. To this end, however, experiment can be of considerable help, as can linear elastic numerical finite-element simulation for which it is now known that relatively small representative volume elements suffice when dealing with linear elastic composites $[8,9,12,16]$. Knowing the relevant modulus expressions for the composite at hand, these can then be adapted with relative ease to derive values for the ratio $C / c$, and in turn to predict the complete monotonic tension/compression elastoplastic composite stress-strain curve, as shown in the preceding discussion. From the tensile curve, in turn, the threedimensional elastoplastic behaviour or the behaviour in unloading situations can then be assessed provided the degree of isotropic to kinematic hardening is estimated; elegant approaches to this end were proposed recently by Suquet and Ponte Castañeda [3,4,29]. Finally, we point out that the variational estimates used here are not free of limitations; the more recent (but more complex) "second-order" variational estimates are better behaved in several ways and can also capture field fluctuations within the phases [4,53-57]. Perhaps these models could similarly be made more "user-friendly".

\section{Conclusion}

- Ponte Castañeda's variational estimate for the prediction of composite non-linear monotonic deformation, equivalent to a "modified" secant modulus method, can be simplified considerably if one decouples elastic from plastic deformation and then assumes, in calculating the plastic strain, that the reinforcement is rigid and the matrix is an incompressible Hollomon power-law von Mises material.

- Making these assumptions, expressions are derived for composites containing spherical elastic inclusions in a Ramberg-Osgood matrix. These give the ratio $C / c$ of the two-phase material to the matrix power-law stress constants. The expressions are simple explicit analytical functions of the matrix power-law exponent and reinforcement volume fraction, also when the linear elastic model that is used is not explicit (as in the DEM and SC schemes).
- Focusing on model composites with a ductile aluminium matrix, varying the power-law exponent, the reinforcement volume fraction, and the elastic contrast, the impact of these simplifications on predicted stress-strain curves is assessed. It is shown that when the MT and the TIHS models are used, the difference between the two schemes (simplified and rigorous models) is small, remaining well below the difference made by the choice of the elasticity model used.

- With the GSC, differences between the simplified and the rigorous methods become significant. This effect is attributed to the fact that the GSC model predicts very stiff composites when the matrix becomes incompressible.

\section{Acknowledgements}

This research was supported by the Swiss National Science Foundation, Project No. 200020-107556. We gratefully acknowledge several interesting discussions on this topic with Dr. Jean-François Despois and Dr. Andreas Rossoll, both at the Laboratory of Mechanical Metallurgy at EPFL.

\section{Appendix A. Calculation of $\mathrm{Clc}$ using the Mori-Tanaka linear elasticity model}

Substituting Eq. (27) into Eq. (21) and using Eq. (26), one obtains

$E_{\mathrm{cs}}=\frac{3 V_{\mathrm{r}}+2}{2\left(1-V_{\mathrm{r}}\right)} \cdot c^{1 / n} \cdot \sigma_{\mathrm{eq}}^{(n-1) / n}$

Eq. (17) becomes

$\sigma_{\mathrm{eq}}=\sqrt{\frac{2}{3 V_{\mathrm{r}}+2}} \cdot \Sigma$

Introducing Eq. (A.2) into Eq. (A.1), one then writes

$\Xi_{\mathrm{pl}}=\frac{\Sigma}{E_{\mathrm{cs}}}=\frac{1-V_{\mathrm{r}}}{\left(\frac{3}{2} V_{\mathrm{r}}+1\right)^{(n+1) / 2 n}} \cdot \frac{\Sigma^{1 / n}}{c^{1 / n}}$

Raising Eq. (A.3) to the power $n$ and rearranging shows that the composite flow stress follows a power-law behaviour with the same exponent $n$ as the matrix (Eq. (20)), with

$\frac{C}{c}=\frac{\left(\frac{3}{2} V_{\mathrm{r}}+1\right)^{(n+1) / 2}}{\left(1-V_{\mathrm{r}}\right)^{n}}$

which is the same as Eq. (28).

\section{References}

[1] Clyne TW, Withers PJ. An introduction to metal matrix composites. Cambridge: Cambridge University Press; 1993.

[2] Suresh S, Mortensen A, Needleman A. Fundamentals of metal matrix composites. Boston (MA): Butterworth-Heinemann; 1993. 
[3] Suquet P, editor. Continuum micromechanics. Vienna: Springer; 1997.

[4] Ponte Castañeda P, Suquet P. In: van der Giessen E, Wu TY, editors. Advances in applied mechanics, vol. 34. San Diego (CA): Academic Press; 1998. p. 171-302.

[5] Masson R, Bornert M, Suquet P, Zaoui A. J Mech Phys Solids 2000;48:1203-27.

[6] Bornert M, Bretheau T, Gilormini P. Homogénéisation en mécanique des matériaux 2: Comportements non linéaires et problémes ouverts (Traité MIM, série alliages métalliques). Paris: Hermès Science Publications; 2000.

[7] Willis JR. Eur J Mech A Solid 2000;19:S165-84.

[8] Drugan WJ, Willis JR. J Mech Phys Solids 1996;44:497-524.

[9] Drugan WJ. J Mech Phys Solids 2000;48:1359-87.

[10] Segurado J, Llorca J. J Mech Phys Solids 2002;50:2107-21.

[11] Böhm HJ, Han W, Eckschlager A. CMES Comput Model Eng Sci 2004;5:5-20.

[12] Gonzalez C, Segurado J, Llorca J. J Mech Phys Solids 2004;52:1573-93.

[13] Böhm HJ, Han W. Model Simul Mater Sci Eng 2001;9:47-65.

[14] Tandon GP, Weng GJ. J Appl Mech Trans ASME 1988;55:126-35.

[15] Nan CW, Yuan RZ. Phys Rev B 1993;48:3042-7.

[16] Segurado J, Llorca J, Gonzalez C. Scr Mater 2002;46:525-9.

[17] Berveiller M, Zaoui A. J Mech Phys Solids 1979;26:325-44.

[18] Nan CW, Clarke DR. J Am Ceram Soc 1997;80:237-40.

[19] Suquet P. In: Suquet P, editor. Continuum micromechanics. Vienna: Springer; 1997. p. 197-264.

[20] Hervé E, Zaoui A. Eur J Mech A Solid 1990;9:505-15.

[21] Suquet P. C R Acad Sci Ser II 1995;320:563-71.

[22] Ponte Castañeda P. J Mech Phys Solids 1991;39:45-71.

[23] Ponte Castañeda P. J Mech Phys Solids 1992;40:1757-88.

[24] Ponte Castañeda P. In: Suquet P, editor. Continuum micromechanics. Vienna: Springer; 1997. p. 131-95.

[25] Li G, Ponte Castañeda P, Douglas AS. Mech Mater 1993;15:279-300.

[26] Li G, Ponte Castañeda P. Appl Mech Rev 1994;47:S77-94.

[27] deBotton G, Ponte Castañeda P. Int J Solids Struct 1993;30:1865-90.

[28] Hu GK. Int J Plasticity 1996;12:439-49.
[29] Suquet P. J Mech Phys Solids 1993;41:981-1002.

[30] Michel JC, Suquet P. Model Simul Mater Sci Eng 1994;2:637-58.

[31] Gonzalez C, Llorca J. J Mech Phys Solids 2000;48:675-92.

[32] Bardella L. Int J Eng Sci 2003;41:741-68.

[33] Moulinec H, Suquet P. Eur J Mech A Solid 2003;22:751-70.

[34] Torquato S. Random heterogeneous materials: microstructure and macroscopic properties. New York (NY): Springer; 2002.

[35] Li G, Ponte Castañeda P. Int J Solids Struct 1993;30:3189-209.

[36] Nemat-Nasser S, Hori M. Micromechanics: overall properties of heterogeneous materials. Amsterdam: North-Holland Elsevier; 1999.

[37] Qiu YP, Weng GJ. J Appl Mech 1992;59:261-8.

[38] Buryachenko VA. Acta Mech 1996;119:93-117.

[39] Mori T, Tanaka K. Acta Metall Mater 1973;21:571-4.

[40] Weng GJ. Int J Eng Sci 1984;22:845-56.

[41] Weng GJ. Int J Eng Sci 1990;28:1111-20.

[42] Budiansky B. J Mech Phys Solids 1965;13:223-7.

[43] Hill R. J Mech Phys Solids 1965;13:213-22.

[44] McLaughlin R. Int J Eng Sci 1977;15:237-44.

[45] Christensen RM, Lo KH. J Mech Phys Solids 1979;27:315-30.

[46] Christensen RM. J Mech Phys Solids 1986;34:639.

[47] Christensen RM. J Mech Phys Solids 1990;38:379-404.

[48] Torquato S. J Mech Phys Solids 1997;45:1421-48.

[49] Torquato S. J Mech Phys Solids 1998;46:1411-40.

[50] Buryachenko VA. In: Marigo JJ, Rousselier G, editors. MECAMAT 93: international seminar on micromechanics of materials. Paris: Eyrolles; 1993. p. 567-78.

[51] Kouzeli M, Weber L, San Marchi C, Mortensen A. Acta Mater 2001;49:3699-709.

[52] Kouzeli M, Weber L, San Marchi C, Mortensen A. Acta Mater 2003;51:6493-6.

[53] Ponte Castañeda P. J Mech Phys Solids 2002;50:737-57.

[54] Ponte Castañeda P. J Mech Phys Solids 2002;50:759-82.

[55] Idiart M, Ponte Castañeda P. C R Mecanique 2005;333:147-54.

[56] Ponte Castañeda P. J Mech Phys Solids 1996;44:827-62.

[57] Idiart M, Moulinec H, Ponte Castañeda P, Suquet P. J Mech Phys Solids 2006;54:1029-63. 